\title{
Quantum mechanics of the two-dimensional circular billiard plus baffle system and half-integral angular momentum
}

\author{
R. W. Robinett* \\ Department of Physics \\ The Pennsylvania State University \\ University Park, PA 16802 USA
}

(Dated: February 1, 2008)

\begin{abstract}
We examine the quantum mechanical eigensolutions of the two-dimensional infinite well or quantum billiard system consisting of a circular boundary with an infinite barrier or baffle along a radius. Because of the change in boundary conditions, this system includes quantized angular momentum values corresponding to half-integral multiples of $\hbar / 2$. We discuss the resulting energy eigenvalue spectrum and visualize some of the novel energy eigenstates found in this system. We also discuss the density of energy eigenvalues, $N(E)$, comparing this system to the standard circular well. These two billiard geometries have the same area $\left(\mathrm{A}=\pi R^{2}\right)$, but different perimeters $(P=2 \pi R$ versus $(2 \pi+2) R)$, and we compare both cases to fits of $N(E)$ which make use of purely geometric arguments involving only $A$ and $P$. We also point out connections between the angular solutions of this system and the familiar pedagogical example of the one-dimensional infinite well plus $\delta$-function potential.
\end{abstract}

PACS numbers: 03.65.Ge, 03.65.Sq

*Electronic address: rick@phys.psu.edu 


\section{Introduction}

Two-dimensional quantum systems can offer a range of interesting new features compared to more familiar 1D systems, including less trivial implementation of boundary conditions leading to energy quantization as well as more sophisticated connections between conserved quantum numbers and symmetries of the system. They can provide model systems to probe the connections between classical and quantum chaos, as in billiard geometries, and they also find proverbial 'real-life' experimental realizations in a number of surface systems, such as atomic corrals. Two-dimensional systems can also provide the first chance to utilize important semi-classical methods such as periodic orbit theory 1, 2], where the detailed structure (specifically the oscillatory component) of the energy level density, $N(E)$, can be very directly connected to the closed classical trajectories in the system. Finally, students may also find connections between solutions of the 2D Schrödinger equation for infinite well or billiard systems and more familiar solutions of wave equations for membranes (drumheads) from mathematical physics courses.

Such 2D quantum infinite well or billiard systems provide practice in the practical application of boundary conditions on the quantum wavefunction arising from purely geometric considerations and a number of example of two-dimensional infinite well systems have been discussed in the literature. The 2D infinite square well [3] can be studied as an example of the product of two separable one-dimensional problems, but also in the context of energy level degeneracy, either due to obvious symmetries or for more subtle reasons [4]. The problem of a $45^{\circ}-45^{\circ}-90^{\circ}$ isosceles triangle billiard [5] can be easily solved using linear combination solutions of the $2 \mathrm{D}$ square well, while the problem of the equilateral $\left(60^{\circ}-60^{\circ}-60^{\circ}\right)$ triangle

billiard 'footprint' has been solved in closed form by a number of authors [6] - [10], using a variety of methods.

The circular infinite well, defined by the potential energy function

$$
V(r, \theta)=V(r)=\left\{\begin{array}{l}
0 \text { for } r<R \\
\infty \text { for } r \geq R
\end{array}\right.
$$

is equivalent to the problem of a classically vibrating circular membrane 11], but has also been considered in detail in the quantum mechanical case [12]. This system can provide students with their first introduction to quantized angular momentum values in a simple context and is the starting point for our discussion. 
In this note, we will extend the analysis of the 'standard' circular well (shown in Fig. 1(a)) to a similar infinite well or quantum billiard system consisting of the same circular 'footprint', but with the addition of an infinitely narrow and infinitely high wall along the $\theta=0$ direction, which we will call a baffle, as shown in Fig. 1(c). The presence of this additional constraint changes the application of the boundary conditions, and hence the allowed quantum numbers, both the quantized values of angular momentum, as well as the energy eigenvalue spectrum. In the next section, we will review the solutions of the full circular well, as well as the related special case of the 'half-circular' well (as shown in Fig. 1(b)), and then use these results to derive the quantum eigensolutions of the circular well plus baffle case. We find that half-integral values (in units of $\hbar$ ) of quantized angular momentum are now allowed and we also visualize some of the novel position-space eigenfunctions

In Sec. 3, we then calculate the distributions of energy levels, $N(E)$, for the full-circular well and circular well plus baffle case, comparing both results to predictions made using purely geometric arguments requiring only knowledge of the area and perimeter of the twodimensional systems, finding excellent agreement with formulae familiar in the mathematical literature. We emphasize the importance and relative straightforwardness of such analyses for 2D billiard systems. We then discuss, in Sec. 4, the similarities between the circle plus baffle system and the familiar pedagogical case of the one-dimensional infinite well plus repulsive $\delta$-function potential [14] - 17], which illustrates how the solutions of the full circle problem are continuously related to the new half-integral angular momentum states. We end by presenting our conclusions and suggesting additional exercises of this type, involving other novel 2D billiard systems which can be analyzed in terms of an energy level density.

\section{Circular well and variations}

We begin by reviewing the derivation of the solutions of the circular infinite well. The two-dimensional, time-independent, free-particle Schrödinger equation, in the relevant polar coordinates, is written in the form

$$
-\frac{\hbar^{2}}{2 \mu}\left(\frac{\partial^{2}}{\partial r^{2}}+\frac{1}{r} \frac{\partial}{\partial r}+\frac{1}{r^{2}} \frac{\partial^{2}}{\partial \theta^{2}}\right) \psi(r, \theta)=E \psi(r, \theta) .
$$

For notational convenience, we have labeled the particle mass as $\mu$, in order to avoid confusion with familiar quantum numbers. We assume a separable solution of the form 
$\psi(r, \theta)=R(r) \Theta(\theta)$, and obtain the angular and radial equations

$$
\frac{d^{2} \Theta_{(m)}(\theta)}{d \theta^{2}}=-m^{2} \Theta_{(m)}(\theta)
$$

and

$$
\frac{d^{2} R(r)}{d r^{2}}+\frac{1}{r} \frac{d R(r)}{d r}-\frac{m^{2}}{r^{2}} R(r)=-k^{2} R(r)
$$

where $k=\sqrt{2 \mu E / \hbar^{2}}$. Initially one can think of $m^{2}$ as simply a separation constant to be determined.

The angular equation has (normalized) solutions of the form

$$
\Theta_{(m)}(\theta)=\frac{1}{\sqrt{2 \pi}} e^{i m \theta}
$$

and the requirement that the solutions be single-valued under redefinitions of the angular variable, namely $\Theta_{(m)}(\theta+2 \pi)=\Theta_{(m)}(\theta)$, implies that

$$
e^{i m(\theta+2 \pi)}=e^{i m \theta} \quad \Longrightarrow \quad m=0, \pm 1, \pm 2, \pm 3, \ldots
$$

giving the familiar integral values of quantized angular momentum. Because of the central nature of the potential, angular momentum is also conserved, with the $\Theta_{(m)}(\theta)$ being the eigenfunctions of $\hat{L}_{z}=(\hbar / i)(\partial / \partial \theta)$ with eigenvalues $m \hbar$.

The resulting equation for the radial component, when written in terms of the variable $z=k r$, becomes,

$$
\frac{d^{2} R(z)}{d z^{2}}+\frac{1}{z} \frac{d R(z)}{d z}+\left(1-\frac{m^{2}}{z^{2}}\right) R(z)
$$

which can be recognized as Bessel's (cylindrical) equation. This has two linearly independent solutions for each value of $|m|$, the so-called regular $J_{m}(z)$ (well-behaved as $z \rightarrow 0$ ) and the singular $Y_{m}(z)$ (divergent as $z \rightarrow 0$ ) solutions. Only the well-behaved $J_{m}(z)$ are used here, with the energy eigenvalues determined by the boundary condition at the circular boundary, namely $J_{m}(k R)=0$. The resulting energy eigenvalues are then given by

$$
E_{\left(m, n_{r}\right)}=\frac{\hbar^{2} k_{\left(m, n_{r}\right)}^{2}}{2 \mu}=\frac{\hbar^{2}}{2 \mu R^{2}}\left[z_{\left(m, n_{r}\right)}\right]^{2}
$$

where $z_{\left(m, n_{r}\right)}$ is the $n_{r}$-th zero of the regular Bessel function $J_{m}(z)$. The number of radial nodes is then given by $n_{r}-1$. The properly normalized radial wavefunctions are given by

$$
\mathcal{J}_{\left(m, n_{r}\right)}(k r) \equiv N_{\left(m, n_{r}\right)} J_{\left(m, n_{r}\right)}(k r) \quad \text { where } \quad\left[N_{\left(m, n_{r}\right)}\right]^{2} \int_{0}^{R}\left[J_{\left(m, n_{r}\right)}(k r)\right]^{2} r d r=1
$$


with $k=z_{\left(m, n_{r}\right)} / R$.

The complete spectrum for the full circular well corresponds to one set of the $m=0$ solutions, but is doubly degenerate for each value of $|m|>0$, since both positive and negative values of $m$ give the same contributions; this corresponds physically to the equivalence of clockwise versus counter-clockwise motions. Some of the low-lying energy eigenvalues (solid lines) are illustrated in Fig. 2, ordered by angular momentum values.

The related case of the half-circular infinite well, shown in Fig. 1(b), can also be solved directly using these results. The angular eigenfunctions in Eqn. (5) can also be written in the (normalized) form

$$
\tilde{\Theta}_{(m)}= \begin{cases}1 / \sqrt{2 \pi} & \text { for } m=0 \\ \cos (m \theta) / \sqrt{\pi} & \text { for } m=1,2,3, \ldots \\ \sin (m \theta) / \sqrt{\pi} & \text { for } m=1,2,3, \ldots\end{cases}
$$

by taking appropriate linear combinations of the $\exp (\operatorname{im} \theta)$ solutions, with the same pattern of degeneracies obvious (namely one $m=0$ state and doubly degenerate $|m| \neq 0$ states.)

The $\sin (m \theta)$ solutions will still satisfy the Schrödinger equation inside the half-circular well, as well as the boundary conditions that the wavefunction vanish along both the $\theta=0$ and $\theta=\pi$ directions. Thus, with a trivial change in normalization (an extra factor of $\sqrt{2}$ to account for the smaller 'footprint'), the $\sin (m \theta)$ solutions are also appropriate for the half-circular well, with the result that the energy eigenvalue spectrum for that problem consists of one copy of the integral $m>0$ energy eigenvalues in Fig. 2. The fact that the 'half-circle' state has roughly half the states of the full circle problem will be elaborated upon in a detailed way in the discussion of energy eigenvalue density in Sec. 3.

Turning now to the case of the circular well plus baffle, as shown in Fig. 1(c), we can also make use of the forms in Eqn. (10), but we must also reinterpret the required boundary condition as imposed by the baffle. The vanishing of the angular wavefunctions along the $\theta=0$ line implies that the $\cos (m \theta)$ combinations (and the $m=0$ state) are not allowed, while the remaining $\sin (m \theta)$ solutions must now also vanish along the $\theta=2 \pi$ line (the same infinite wall baffle) which implies that we must have

$$
\sin (2 \pi m)=0 \quad \Longrightarrow \quad m=\frac{1}{2}, 1, \frac{3}{2}, 2, \frac{5}{2}, \ldots
$$

and half-integral values of the angular momentum quantum number, $m$, are now allowed. The integral $m=1,2,3, \ldots$ solutions from Eqn. (10) are still allowed, this time with the 
same normalization as for the full circular well, but there is a new class of angular (and hence radial solutions) characterized by $m=(2 j+1) / 2=1 / 2,3 / 2,5 / 2, \ldots$.

As we will see in detail in Sec. 4, the angular eigenfunctions corresponding to half-integral values of $m$ correspond to the even functions of $\theta$ (about $\theta=0$ ), so we can also write these solutions in the form

$$
\tilde{\Theta}_{(m)}(\theta)=\frac{1}{\sqrt{\pi}} \sin (m|\theta|) \quad \text { for } m=(2 j+1) / 2=1 / 2,3 / 2, \ldots
$$

if we choose to define them over the angular interval $(-\pi,+\pi)$. The first four lowest lying angular solutions corresponding to $m=1 / 2,1,3 / 2$, and 2 are shown in Figs. 3(a), (b), (c), and (d) respectively, both over the interval $(0,2 \pi)$ and $(-\pi,+\pi)$, illustrating both the symmetry as well as the 'cusp' in $\tilde{\Theta}_{(m)}(\theta)$ at $\theta=0$, induced by the infinite wall, for the $m=(2 j+1) / 2$ values. Because of the additional 'angular barrier', the potential is no longer purely central, and angular momentum is not conserved. The $\tilde{\Theta}_{(m)}(\theta)$ are, however, still eigenfunctions of $\hat{L}_{z}^{2}$, with eigenvalues given by $(m \hbar)^{2}$; this corresponds classically to the fact that particles would 'rebound' from the baffle, reversing direction, and hence the sign of angular momentum, but keeping the same magnitude of $L_{z}$.

The resulting energy eigenstates, $\psi(r, \theta)$, for the integral $m$ values are identical to the odd-parity states of the full circular well (since they already vanish completely along the entire $\pm x$ axis), but the (normalized) wavefunctions for the half-integral $m=(2 j+1) / 2$ angular momentum states states are now given by

$$
\psi(r, \theta)=\mathcal{J}_{(m=j+1 / 2)}(k r) \tilde{\Theta}_{(2 j+1) / 2}(\theta) \quad \text { with } \quad k=z_{\left(j+1 / 2, n_{r}\right)} / R
$$

We can use the well-known connection between the (cylindrical) Bessel functions, $J_{m}(z)$, and the spherical Bessel functions, $j_{m}(z)$ (obtained from the 3D version of the free particle Schrödinger equation [18]) to write the half-integral solutions in the form

$$
j_{m}(z)=\sqrt{\frac{\pi}{2 z}} J_{m+1 / 2}(z)
$$

Thus, half of the solutions of the 2D circular well plus baffle, those corresponding to halfintegral angular momentum, can actually be described by integral angular momentum solutions, but of the corresponding 3D problem. For example, the two lowest $m$-value spherical 
Bessel functions are given by

$$
\begin{aligned}
& j_{0}(z)=\frac{\sin (z)}{z} \\
& j_{1}(z)=\frac{\sin (z)}{z^{2}}-\frac{\cos (z)}{z} .
\end{aligned}
$$

The energy eigenvalues for the $m=1 / 2$ case then correspond to the zeros of the $3 \mathrm{D} j_{0}(z)$ function, namely $z=n_{r} \pi$, with $n_{r}=1,2, \ldots$ with the result that the $m=1 / 2$ energies are given by the very simple formula

$$
E_{\left(m=1 / 2, n_{r}\right)}=\frac{\hbar^{2} \pi^{2} n_{r}^{2}}{2 \mu R^{2}}
$$

We illustrate the energy eigenvalues corresponding to half-integral values of $m$ in Fig. 2, where they nicely interpolate between the standard integral $m$ results. The number of states for the circular well plus baffle case is then, in some sense, exactly the same as that for the standard circular well, and we will in fact argue in Sec. 4, that the half-integral angular momentum states correspond very directly to the even-parity integral- $m$ states of the full circle, transforming into them as the effect of the baffle is slowly added.

We then plot in Figs. 4 and 5 the position-space probability density $\left(|\psi(r, \theta)|^{2}\right)$ for the two lowest-lying energy eigenstates for the $m=1 / 2$ and $m=3 / 2$ states respectively, which can be compared to the more familiar looking (drumhead-like) case of $m=1$ shown in Fig. 5.

For all cases with $m>1 / 2$, because of the fairly obvious symmetries of the solutions, (with $s=2 m$ 'spokes' along nodal lines at $\theta=2 \pi / s$ ), the expectation values of the position variables can be easily shown to vanish, namely, $\langle x\rangle=\langle y\rangle=0$ for all $n_{r}$ values when $m>1 / 2$. For the special case of $m=1 / 2$, however, the expectation value of

$\left\langle\tilde{\Theta}_{(1 / 2)}|\cos (\theta)| \tilde{\Theta}_{(1 / 2)}\right\rangle_{m=1 / 2}=-1 / 2$ combines with the simple form of the radial solutions from Eqn. (15) to give $\left\langle\mathcal{J}_{(1 / 2)}(k r)|r| \mathcal{J}_{(1 / 2)}(k r)\right\rangle=R / 2$ to yield $\langle x\rangle_{\left(m=1 / 2, n_{r}\right)}=-R / 4$ and this asymmetry is also obvious from Fig. 4.

\section{Energy level density and completeness of the energy eigenstates}

The calculation of the number of normal modes of solutions of the wave equation (scalar, electromagnetic, etc.) in 2-D or 3-D cavities is an important problem in a number of branches of physics, with many discussions building on early work by Weyl. Students are perhaps 
most familiar with this problem in the context of the derivation of the Planck formula for blackbody radiation for a simple 3D cubical cavity which is often included in the modern physics curriculum, but many interesting mathematical results exist for rather general twodimensional shapes.

A standard reference text [19] on theoretical physics shows that the number of normal mode wavenumbers in the range $(k, k+d k)$ for a $2 \mathrm{D}$ shape of area $A$ and perimeter $P$ is given by

$$
d N(k)=\left[\frac{A}{2 \pi} k-\frac{P}{4 \pi}\right] d k
$$

which upon integration gives

$$
N(k)=\frac{A}{4 \pi} k^{2}-\frac{P}{4 \pi} k
$$

or, in the context of solutions of the Schrödinger equation of relevance here,

$$
N(E)=\frac{A}{4 \pi}\left(\frac{2 \mu}{\hbar^{2}} E\right)-\frac{P}{4 \pi} \sqrt{\frac{2 \mu}{\hbar^{2}} E} .
$$

The 'experimental' energy spectrum is, of course, a discontinuous 'staircase' function of the form

$$
N(E)=\sum_{i} \theta\left(E-E_{i}\right)
$$

and so the Weyl-like result of Eqn. (201) will be an approximation to a smoothed out version of the 'data'.

This type of analysis can be extended [20] to include an additional constant (and hence subleading) term which arises from the consideration of such geometrical effects as corners, curvature, and the connectivity of the 2D domain. Perhaps more importantly, the oscillatory behavior of $N(E)$ is the central theme of periodic orbit theory [1], 2] and simple pedagogical examples of this have been given 21] for the square, circular well, and half circle 'footprints'.

The result of Eqn. (20) can be rather easily tested on familiar 2D billiard/infinite well systems such as the square or rectangular wells, as well as both the $45^{\circ}$ isosceles and $60^{\circ}$ equilateral triangles, all because of their extremely simple energy eigenvalue formulae (consisting of simple quadratic powers of two integral quantum numbers.) In fact, two studies of the equilateral triangle billiard [6], [7] made use of this relation as a cross-check on the completeness of the energy eigenstates they derived, confirming that their calculated energy spectrum saturated the Weyl-like prediction for $N(E)$. 
For the cases we consider here, we wish to compare the theoretically predicted spectrum for $N(E)$ against the geometric 'fit' of Eqn. (20). From the differing 'footprints' for the geometries we have considered, the relevant area $(A)$ and perimeter $(P)$ values are given by

\begin{tabular}{|l|l|r|}
\hline shape & $A$ & $P$ \\
\hline full circular well & $\pi R^{2}$ & $2 \pi R$ \\
\hline half circular well & $\pi R^{2} / 2$ & $(2+\pi) R$ \\
\hline well plus baffle & $\pi R^{2}$ & $(2 \pi+2) R$ \\
\hline
\end{tabular}

so that the full circular well and the well plus baffle have the same area, but differing perimeters (due to the 'intrusive' baffle.) Note that the effective perimeter for the well plus baffle case is $2 R$ larger than $2 \pi R$ due to the fact that both the 'top' and the 'bottom' of the baffle are inside the well, each adding a factor of $R$.

We can easily collect (using numerical calculations of the required zeros of various order Bessel functions) large numbers of the low-lying energy eigenvalues for the cases we consider and we plot the resulting $N(E)$ versus $E$ using the first hundred or so lowest $E$ values for the three cases above in Fig. 7(a), as the three 'staircase' functions shown there. The smooth curves are the predictions of Eqn. (20), while the two dotted curves are the predictions using only the area $(A)$ terms which are clearly not a very good fit by themselves. The correspondence to the observed energy spectra is extremely good, and the clear difference between the full circle and half circle cases is obvious. A smaller region (shown as a dashed box) is enlarged on the right in Fig. 7(b) and there one can here easily see the nice distinction between the full circle case (upper data and dashed curve) and the circle plus baffle (lower data and solid curve) indicating that the effective values of $A$ and $P$ used above are correct. This case is especially interesting since it's one of the few for which the 'footprint' area is naturally the same, while the perimeter is different. The case of the $2 \mathrm{D}$ infinite square well $(L \times L)$ or an equal area rectangular well $(f L \times L / f)$ with different perimeters is another such simple example.

We wish to especially emphasize how this type of analysis can be rather easily applied to a wide variety of $2 \mathrm{D}$ billiard systems, perhaps with an eye towards introducing students to numerical methods, and eventually to the interesting topic of periodic orbit theory. We also note that the discussion can be made more quantitative by applying least square fits to the $N(E)$ versus $E$ data used in Fig. 7, using a functional form $N(E)=a E+b \sqrt{E}$, to obtain 
numerical values to compare directly to the results of Eqn. (20), with good agreement being obtained using only a few hundred low-lying states.

\section{Relationship to 1D infinite well plus $\delta$ function prob- lem}

In order to better understand the structure of the half-integral angular wavefunctions derived above, and to make connection with a familiar one-dimensional quantum system, we wish to model the effect of introducing the baffle wall and continuously increasing its height. This approach is, then, very similar to the often discussed [14] - 17] problem of a 1D infinite square well with a repulsive $\delta$-function potential placed at the center. We will briefly review the methodology and results of that problem, and then apply it to case of the circular baffle.

As an example of how the additional of a singular potential of arbitrary 'strength' can change the energy level structure of a simple system, we consider a symmetric infinite well potential with walls at $(-L,+L)$, which has even- and odd-parity energy eigenfunctions and eigenvalues given by

$$
\begin{array}{cccc}
\psi_{(n)}^{(+)}(x)=\frac{1}{\sqrt{L}} \cos \left(\frac{(2 n-1) \pi x}{2 L}\right) & \text { with } & E_{(n)}^{(+)}=\frac{\hbar^{2} \pi^{2}(2 n-1)^{2}}{8 \mu L^{2}} \\
\psi_{(n)}^{(-)}(x)=\frac{1}{\sqrt{L}} \sin \left(\frac{(2 n) \pi x}{2 L}\right) & \text { with } & E_{(n)}^{(-)}=\frac{\hbar^{2} \pi^{2}(2 n)^{2}}{8 \mu L^{2}}
\end{array}
$$

where once again we denote the particle mass by $\mu$.

We then introduce a singular, repulsive potential of the form $V(x)=\lambda \delta(x)$ to the center of the well and ask how the energy eigenvalues and eigenfunctions are changed. The odd parity $\psi_{(n)}^{(-)}(x)$, which have a node at $x=0$, are unaffected by the addition of $V(x)$, whatever the strength of the singularity, $\lambda$. The even parity solutions are changed and we can write a very general even solution in the form

$$
\psi_{(\lambda)}^{(+)}(x)= \begin{cases}A \cos (k x)+B \sin (k x) & \text { for } 0 \leq x \leq+L \\ A \cos (k x)-B \sin (k x) & \text { for }-L \leq x \leq 0\end{cases}
$$

The boundary conditions at either wall (where $\psi$ must vanish) and at the singularity (where $\psi^{\prime}(0)$ is discontinuous) are

$$
\psi_{(\lambda)}(-L)=\psi_{(\lambda)}(+L)=0 \quad \text { and } \quad \psi_{(\lambda)}^{\prime}\left(0_{+}\right)-\psi_{(\lambda)}^{\prime}\left(0_{-}\right)=\frac{2 \mu \lambda}{\hbar^{2}} \psi_{(\lambda)}(0)
$$


and give the relations

$$
A \cos (k l)+B \sin (k L)=0 \quad \text { and } \quad 2 B k=\frac{2 \mu \lambda}{\hbar^{2}} A
$$

which combine to yield the energy eigenvalue condition

$$
\lambda\left(\frac{\mu L}{\hbar^{2}}\right)=-\frac{k L \cos (k L)}{\sin (k L)} .
$$

For the case of $\lambda=0$ (no additional $\delta$ perturbation), the solutions are given by $k L=$ $(2 n-1) \pi / 2$ and the standard $\psi_{(n)}^{(+)}(x)$ states are reproduced. In the limit of $\lambda \rightarrow+\infty$, however, a constant horizontal line of $\lambda\left(\mu L / \hbar^{2}\right)$ cuts the right hand side of Eqn. (27) at $k L=n \pi$, so that the even energy eigenvalue solutions approach those of the (unchanged) odd parity solutions from below, namely $E_{(\lambda)}^{(+)}(\lambda \rightarrow \infty) \rightarrow E_{(n)}^{(-)}$and the energy spectrum is now doubly degenerate for each $n$ value.

For the case of the baffle added to the standard circular well, we will model the effect of continuously 'turning on' the baffle by re-writing the angular Schrödinger equation in Eqn. (3) in the form

$$
-\frac{d^{2} \Theta_{(m)}(\theta)}{d \theta^{2}}+g \delta(\theta)=m^{2} \Theta_{(m)}(\theta)
$$

with the angles defined over the symmetric interval $(-\pi,+\pi)$. The odd parity (in $\theta)$ angular $\sin (m \theta)$ solutions of Eqn. (10) are unaffected by the additional repulsive $\delta$ interaction, while we can write the even solutions, for arbitrary values of $g$, in a very similar form to that in Eqn. (24), namely

$$
\Theta_{(m)}^{(+)}(\theta)= \begin{cases}A \cos (m \theta)+B \sin (m \theta) & \text { for } 0 \leq \theta \leq+\pi \\ A \cos (m \theta)-B \sin (m \theta) & \text { for }-\pi \leq \theta \leq 0\end{cases}
$$

The relevant boundary conditions are now on the continuity of $\Theta^{\prime}(\theta)$ at $\theta= \pm \pi$ and on the correct discontinuity at $\theta=0$ which give

$$
2 B m \cos (m \pi)=2 A m \sin (m \pi) \quad \text { and } \quad 2 m B=g A
$$

respectively. These combine to give the condition for the quantized angular momentum quantum numbers, $m$, as

$$
g=\frac{2 m \sin (m \pi)}{\cos (m \pi)} \equiv f(m)
$$

We plot the right-hand-side of Eqn. (31) in Fig. 8 and note that solutions of this eigenvalue problem once again correspond to the intersections of horizontal lines of constant $g$ with 
the various branches of $f(m)$. For $g=0$, namely the case of the standard circular well without the baffle, the intersections are at integral values of $m$, including $m=0$, and the even and odd angular wavefunctions are doubly degenerate (except for $m=0$ ) as noted above. For the case of $g \rightarrow+\infty$, the intersections arise at half-integral values of $m$, so that $m=1 / 2,3 / 2, \ldots$ and the angular solutions are no longer degenerate. One can, in fact, write closed-form expressions for the (appropriately normalized) even parity angular eigenfunctions in the form

$$
\tilde{\Theta}_{(m)}^{(+)}(\theta)=\frac{\cos \left(m|\theta|-\phi_{m}\right)}{\sqrt{\pi\left(1+\sin (2 m \pi) \cos \left(2 \phi_{m}\right) / 2 m \pi\right)}}
$$

where $\phi_{m}$ is given by $\tan \left(\phi_{m}\right)=g / 2 m$ and $m$ is given by the solutions of Eqn. (31). This form actually gives the correct normalization for both the $g \rightarrow 0$ limit of integral angular momentum values (both $m=0$ and $m>0$ ) and the $g \rightarrow+\infty$ limit of half-integral values.

\section{Conclusions and discussion}

We have analyzed the circular well plus baffle system in detail, making use of the Weyl-like energy eigenvalue distribution of Eqn. (20) for two-dimensional quantum billiard systems. The system studied here can also be approached as the limiting case of a circular 'slice' or 'wedge' potential [22]. A circular infinite well, where the angle subtended by the billiard is given by $\Phi=(1+f) \pi$, can interpolate between the half-circle case (when $f=0$ ) and the well circular well plus baffle case (when $f=1)$. The boundary conditions $(\sin (m \Phi)=0)$ imply that the angular momentum values must satisfy $m=n /(1+f)$ for integral $n$ and the one can still easily find the zeroes of $J_{(m=n /(1+f))}(z)$ using standard mathematical packages. Using this type of data, one can confirm that the energy eigenstate distribution, $N(E)$, still tracks the result of Eqn. (20), with continuously varying areas and perimeters now given by $A_{f}=(1+f) \pi R^{2}$ and $P_{f}=(2+(1+f) \pi) R$. One can also consider $-1<f<0$ to handle such cases as the 'quarter-circle' billiard and even smaller slices.

Another related case which can be analyzed using these methods is the annular infinite well or billiard [23], 24], where a second concentric infinite barrier at $R_{\text {in }}=f R$ (with $0<f<1)$ is added, and the particle is confined to the radial region $R_{\text {in }}=f R<r<R$. The angular solutions (with or without a baffle) are easily obtained as above, while the radial eigenfunctions are linear combinations of both cylindrical Bessel functions,

$$
R(r)=\alpha J_{m}(k r)+\beta Y_{m}(k r)
$$


where the divergent $Y_{m}(k r)$ is included since the singular $r=0$ point is explicitly excluded. Imposing the boundary condition that $R\left(R_{\text {in }}=r R\right)=0$ as well as vanishing at the outside boundary, gives the eigenvalue condition

$$
J_{m}(k r) Y_{m}(f k R)-J_{m}(f k R) Y_{m}(k R)=0
$$

and one can once again evaluate $N(E)$ and compare it to Eqn. (20) using $A_{f}=\pi R^{2}\left(1-f^{2}\right)$ and $P_{f}=2 \pi R(1+f)$ with good agreement. Interested students can find a variety of relatively simple 2D quantum billiard geometries for which such an analysis is fairly straightforward.

\section{Acknowledgments}

This work was supported in part by the National Science Foundation under Grant DUE9950702 .

[1] Gutzwiller M 2001 The Interplay Between Classical and Quantum Mechanics (College Park, MD: American Association of Physics Teachers)

[2] Brack M and Bhaduri 1997 Semiclassical Physics (Reading MA: Addison-Wesley)

[3] See, e.g., Liboff R L 1980 Introductory Quantum Mechanics (Reading MA: Addison Wesley) pp 294-298

[4] Leyvraz F, Frank A, Lemus R and Andrés M V 1997 Accidental degeneracy in a simple quantum system: A new symmetry group for the particle in an impenetrable square-well potential Am. J. Phys. 65 1087-1094

[5] Li W K 1984 A particle in an isosceles right triangle J. Chem. Edu. 611034

[6] Jung C 1980 An exactly soluble three-body problem in one-dimension Can. J. Phys. 58 719-728

[7] Richens P J and Berry M V 1981 Pseudointegrable systems in classical and quantum mechanics Physica 2D 495-512

[8] A version of this problem is discussed by Mathews J and Walker R L 1970 Mathematical Methods of Physics, 2nd edn (Menlo Park CA: Benjamin) pp 237-239

[9] Li W K and Blinder S M 1987 Particle in an equilateral triangle: Exact solution of a nonseparable problem J. Chem. Educ. 64 130-132 
[10] Doncheski M A and Robinett R W 2002 Quantum mechanical analysis of the equilateral triangle billiard: periodic orbit theory and wave packet revivals Ann. Phys. (New York) 299 208-227

[11] See, e. g., Ref. 8] pp 231-233

[12] Robinett R W 1996 Visualizing the solutions for the circular infinite well in quantum and classical mechanics Am. J. Phys. 64 440-445. See also Ref. [13].

[13] Bressanini D and Ponti A 1998 Angular momentum and the two-dimensional free particle J. Chem. Ed. 75 916-917

[14] Gettys W E 1973 Quantum theory of a square well plus delta function potential Am. J. Phys. $41670-677$

[15] Lapidus I R 1982 One-dimensional hydrogen atom in an infinite square well Am. J. Phys. 50 $563-564$

[16] Lapidus I R 1985 Particle in a square well with a $\delta$-function potential Am. J. Phys. 55 172-174

[17] Vugalter G A, Das A K, and Sorokin V A 2002 Revivals in an infinite square well in the presence of a $\delta$ wall Phys. Rev. A 66012104 (7 pages)

[18] See, e. g. Gasiorowicz S 1995 Quantum Physics 2nd edn. (New York: Wiley) pp178-180

[19] Morse P M and Feshbach H 1953 Methods of Theoretical Physics: Part I (New York: McGrawHill) pp 759-763

[20] Baltes H P and Hilf E R 1976 Spectra of Finite Systems - Review of Weyl's Problem: The eigenvalue distribution of the wave equation for finite domains and its application to physics of small systems (Mannheim: B. I. Wissenschaftsverlag)

[21] Robinett R W 1997 Visualizing classical periodic orbits from the quantum energy spectrum via the Fourier transform: Simple infinite well examples Am. J. Phys 65 1167-1175

[22] Robinett R W 1998 Periodic orbit theory of a continuous family of quasi-circular billiards J. Math. Phys. 39 278-298

[23] Robinett R W 1999 Periodic orbit theory analysis of the circular disk or annular billiard Am. J. Phys. 67 67-77

[24] Robinett R W 1998 Energy eigenvalues and periodic orbits for the circular disk or annular infinite well Surf. Rev. Lett. 5 519-526 


\section{Figure Captions}

Fig. 1. Geometric 'footprints' for the (a) circular infinite well (or billiard), (b) the half-circular well, and (c) the circular well with baffle, studied here.

Fig. 2. Energy spectrum versus quantized angular momentum values relevant for the circular, half-circular, and circular well with baffle cases. Energy values (in units of $\hbar^{2} / 2 \mu R^{2}$ ) for integral values of angular momentum $(m=0,1,2, \ldots)$ are shown as solid, while results for half-integral $(m=1 / 2,3 / 2, \ldots)$ are shown as dashed. The spectrum for the full circular well consists of one copy of the $m=0$ values and two sets of the integral $m>0$ values, while for the half-circular well, one set of the integral $m>0$ values gives the entire spectrum. For the circular well plus baffle case, one set of both the integral and half-integral values with $m>0$ constitute the entire energy spectrum.

Fig. 3. Angular wavefunctions, $\tilde{\Theta}_{(m)}(\theta)$ versus $\theta$, for half-integral and integral values of quantized angular momentum for (a) $m=1 / 2$, (b) $m=1$, (c) $m=3 / 2$, and (d) $m=2$, over the ranges $(0,2 \pi)$ and $(-\pi,+\pi)$. The 'cusps' at $\theta=0$ for the half-integral cases, induced by the infinite baffle along $\theta=0$, are evident.

Fig. 4. Normalized position-space probability densities, $|\psi(r, \theta)|^{2}$, for the two lowest-lying $m=1 / 2$ energy eigenstates $\left(n_{r}=1\right.$ on top and $n_{r}=2$ at the bottom).

Fig. 5. Same as Fig. 4, but for the two lowest-lying $m=3 / 2$ states.

Fig. 6. Same as Fig. 4, but for the two lowest-lying $m=1$ states. These states are also solutions for the standard full circular well.

Fig. 7. Energy eigenvalue distribution, $N(E)$ versus $E$, for the full circle and circle plus baffle (top two curves in (a)) and half-circle case (bottom curve in (a)). The dashed and solid lines are the Weyl-like predictions of Eqn. (20), while the dotted lines are the result of only using the first 'area' term. The dashed square part of (a) is expanded at the right in (b) to show the fine details and the clear differences between the full circle (top, dashed line) and circle plus baffle (bottom, solid line) cases.

Fig. 8. Plot of the 'eigenvalue' condition of Eqn. (31) versus $m$ for even-parity angular wavefunctions. Horizontal lines of constant $g$ cut the curve at solutions of the eigenvalue 
condition for allowed values of $m$. For $g=0$ (corresponding to no baffle), the allowed eigenvalues are $m=0,1,2, \ldots$ as expected, while for $g \rightarrow+\infty$, the intersections are at half-integral values given by $m=1 / 2,3 / 2, \ldots$ corresponding to the circle plus baffle case. 


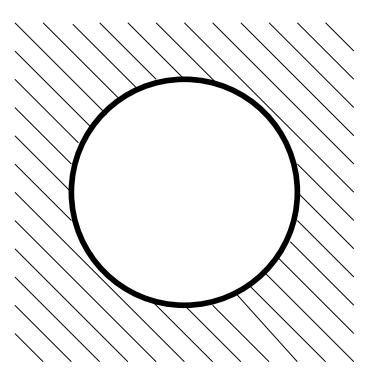

(a)

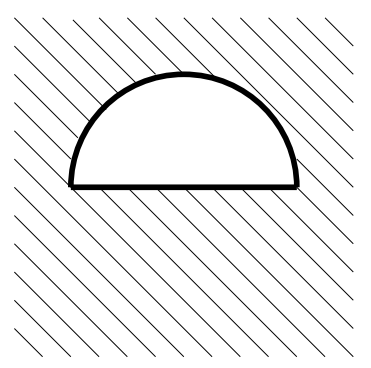

(b)

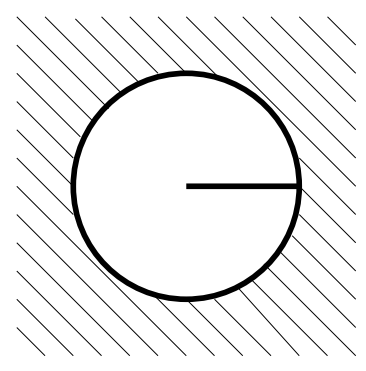

(c)

FIG. 1: 


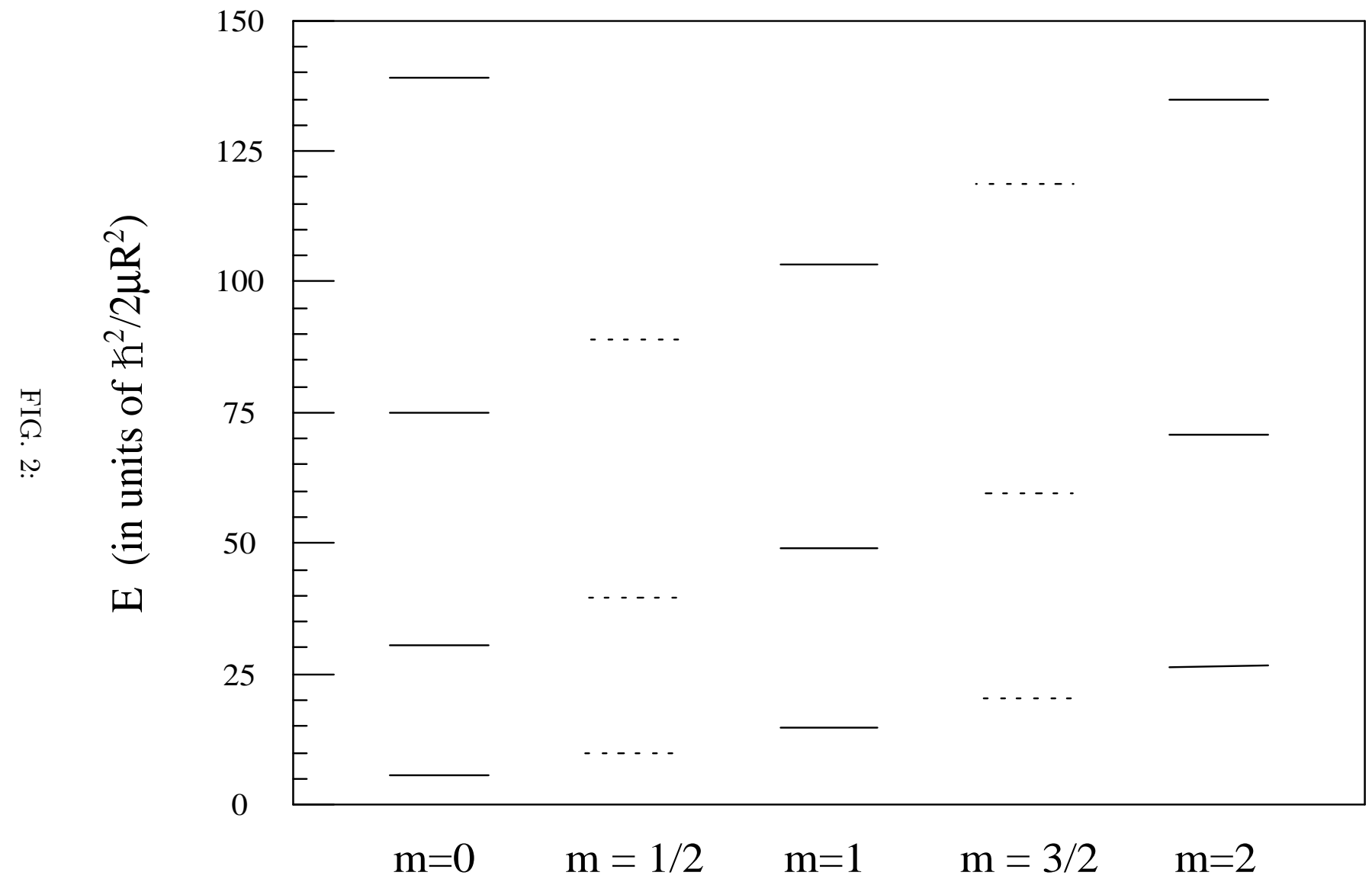


(a)

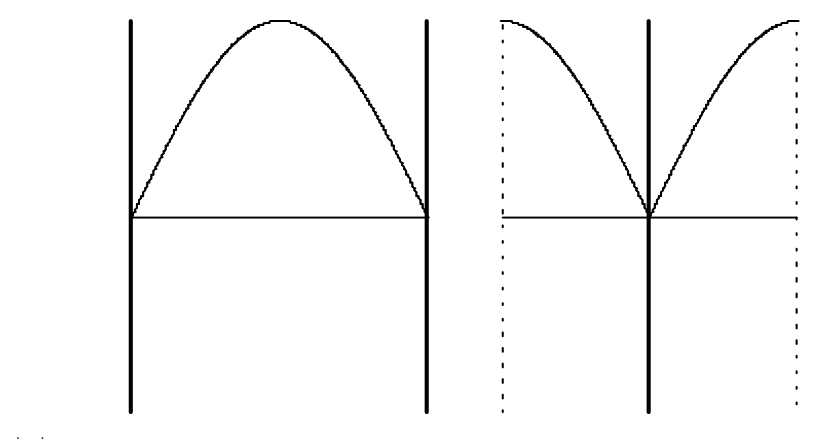

몸

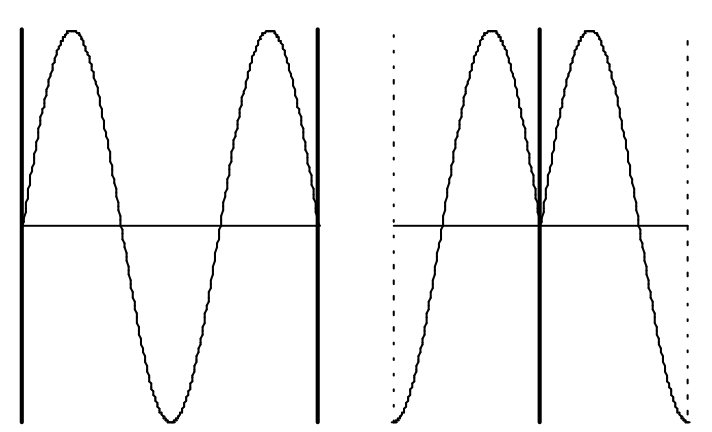

(c) (b)
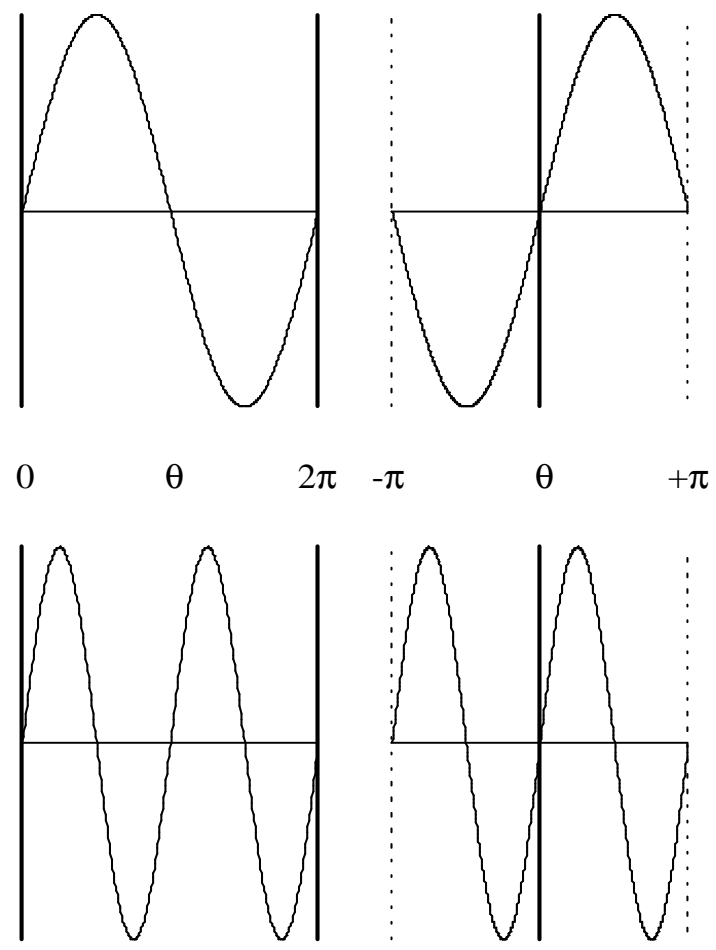

(d) 

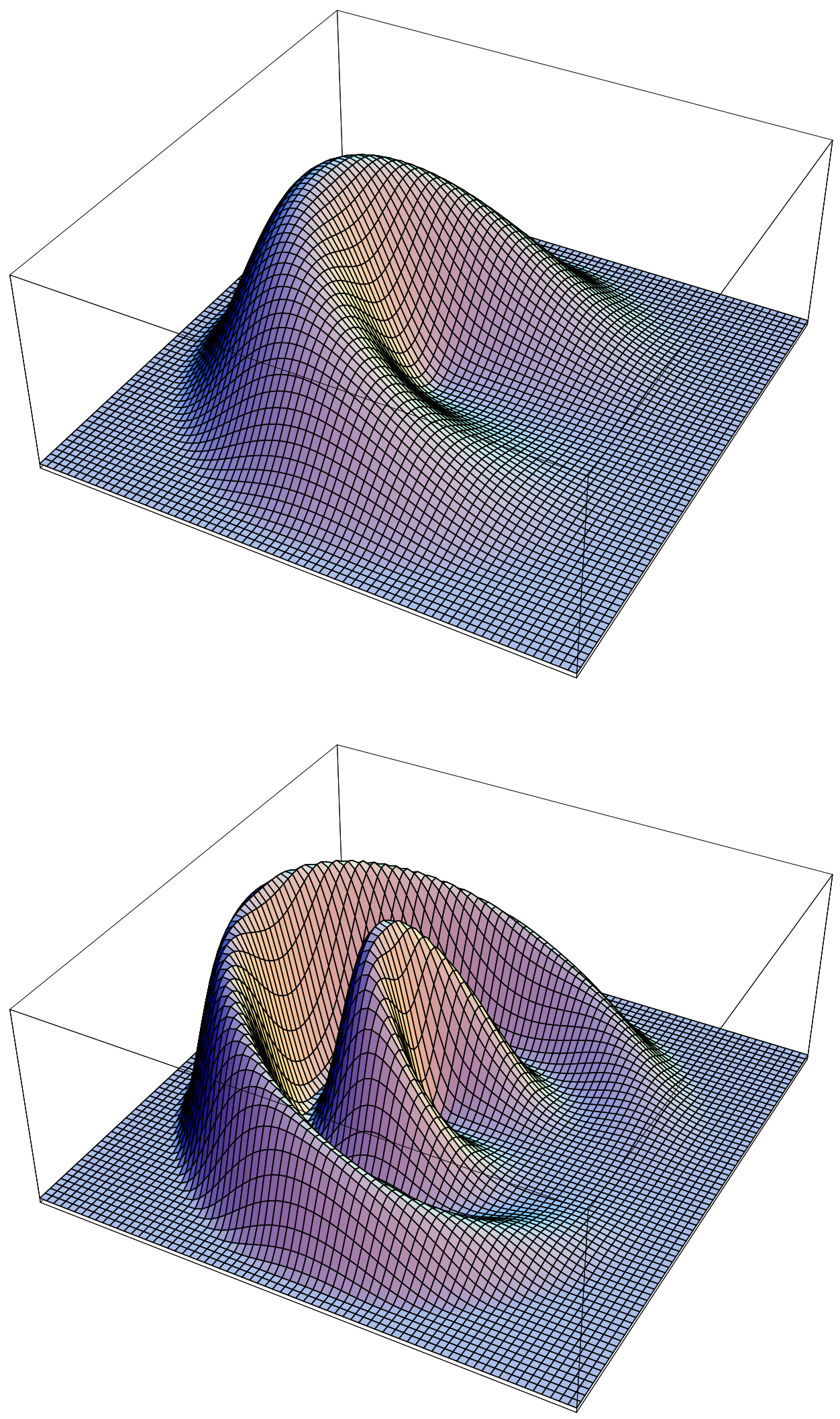

FIG. 4: 

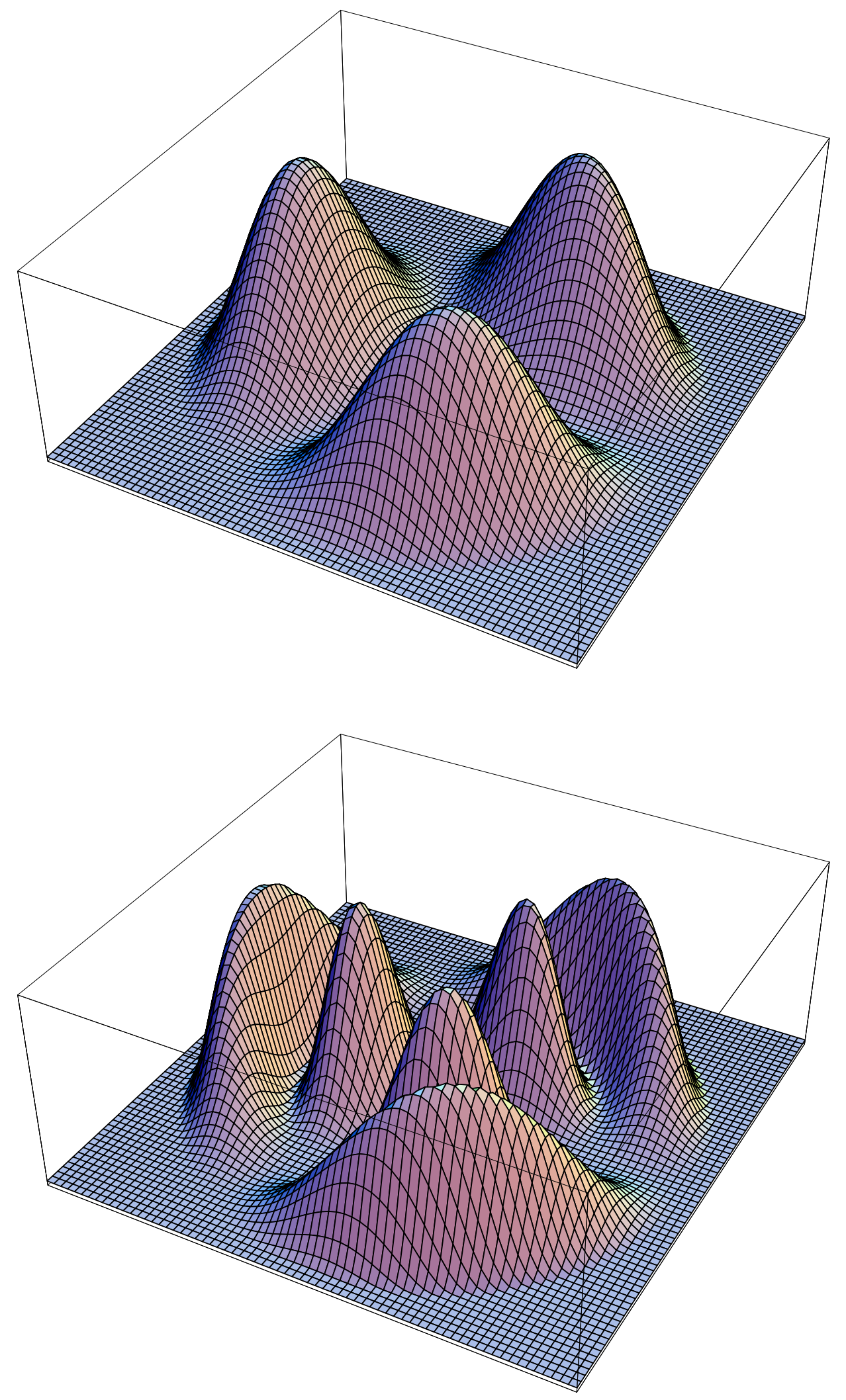

FIG. 5: 

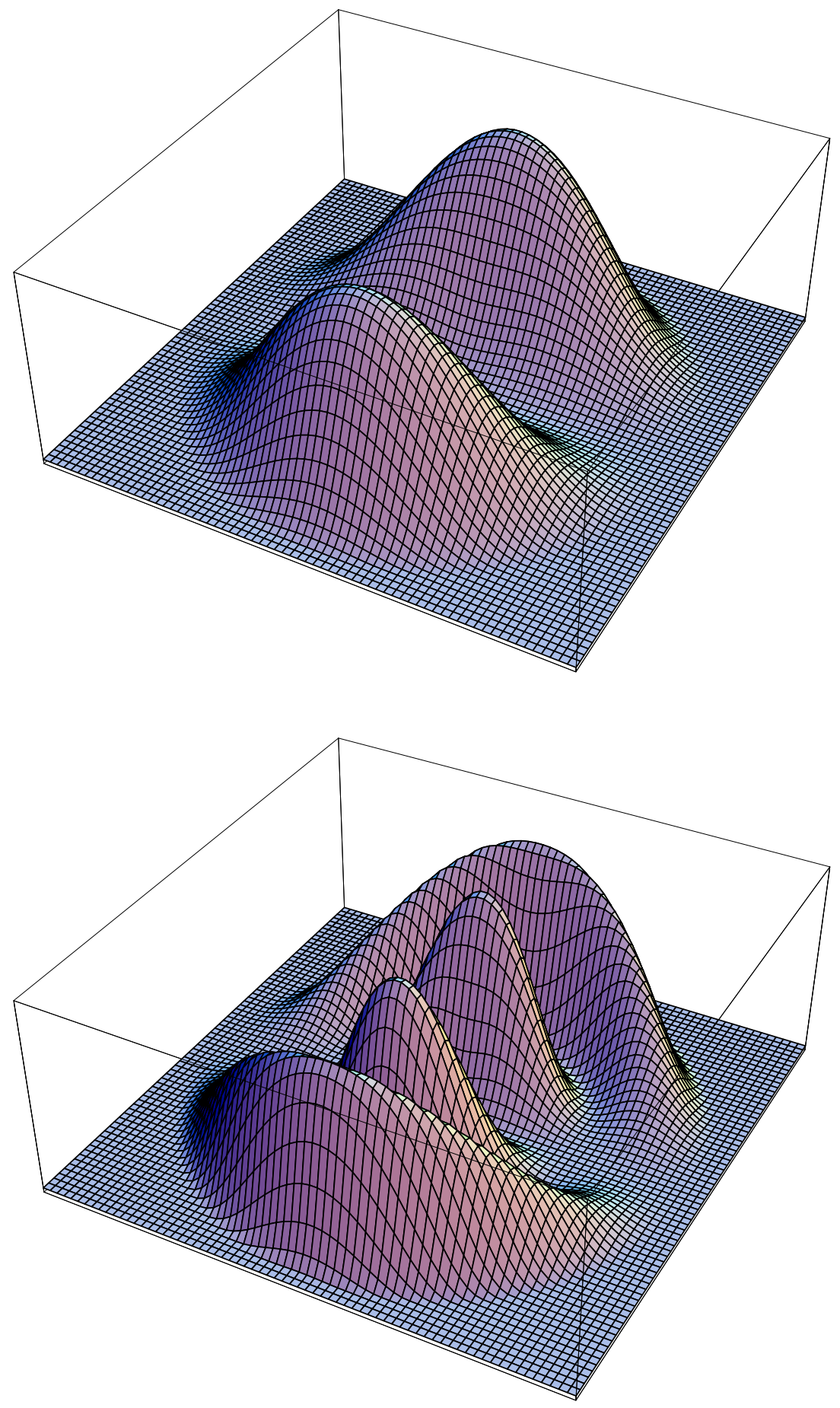

FIG. 6: 
(a)

(b)

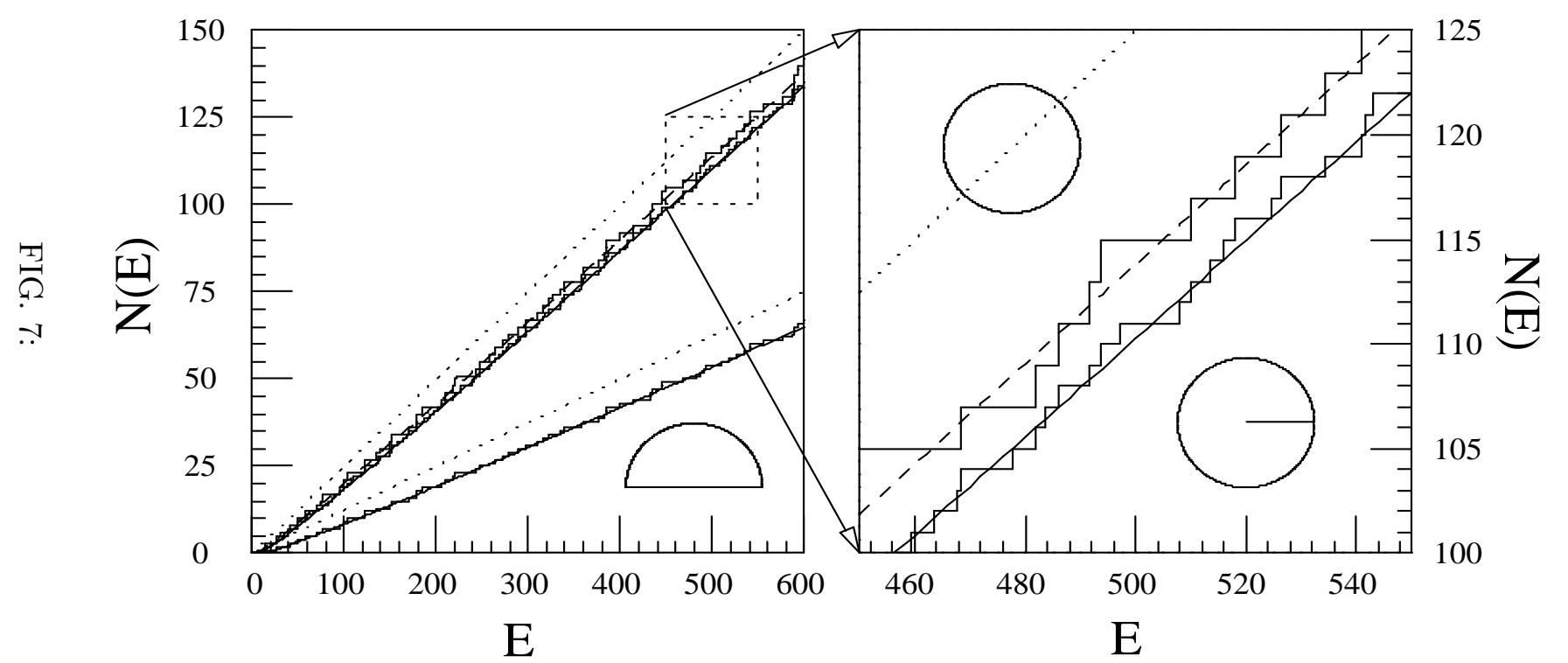




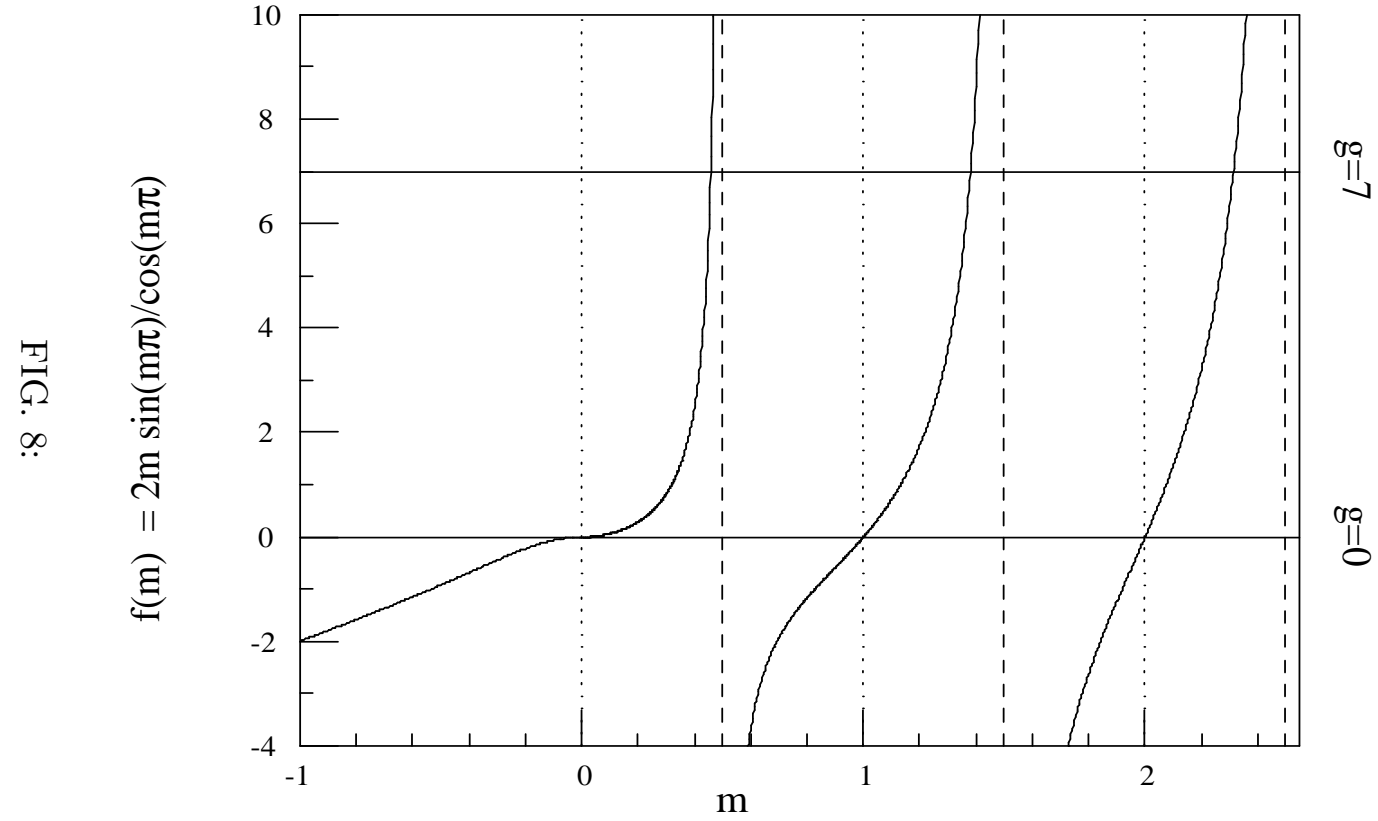

